\title{
Characteristics of Children Referred for Drainage of Subcutaneous Soft Tissue Infections
}

\author{
Kristin L. Long, Leah Gilligan, Kasey M. Cox, John M. Draus Jr.* \\ Department of Surgery, Division of Pediatric Surgery, University of Kentucky Lexington, Kentucky, USA. \\ Email: *jmdr226@uky.edu \\ Received May $4^{\text {th }}, 2013$; revised June $4^{\text {th }}, 2013$; accepted June $16^{\text {th }}, 2013$ \\ Copyright (c) 2013 Kristin L. Long et al. This is an open access article distributed under the Creative Commons Attribution License, \\ which permits unrestricted use, distribution, and reproduction in any medium, provided the original work is properly cited.
}

\begin{abstract}
Purpose: Severe skin and soft tissue infections in children are a common cause of hospital admission. Surgical drainage is often required for definitive therapy. Our aim was to review common characteristics of children requiring surgical incision and drainage (I\&D) for subcutaneous abscesses. Methods: IRB approval was obtained for a retrospective chart review of children requiring I\&D of subcutaneous abscesses between July 2006 and June 2011. Data points included patient demographics, abscess location, surgical procedure, microbiology cultures, and hospital length of stay. Results: Surgical I\&D were performed on 1042 children, and 781 patients (75\%) were admitted to the surgical service. The average age was 3.4 years. 605 patients (58\%) were female. 637 abscesses $(61 \%)$ were located in the groin/buttock/perineum area. Methicillin-resistant Staphylococcus aureus (MRSA) was identified in $70 \%$ of the culture specimens. The average length of stay was 2.8 days. The rate of recidivism was $10.9 \%$. Conclusions: Soft tissue infections requiring hospital admission and $I \& D$ are common in the pediatric population. The vast majority of these are due to MRSA infections. Infections requiring drainage most frequently occurred in the diaper area of girls less than 3 years old. A significant number of children have recurrent skin infections and represent an important patient subpopulation.
\end{abstract}

Keywords: Soft Tissue Infections; Methicillin-Resistant Staphylococcus aureus; Incision and Drainage; Children

\section{Introduction}

Skin and soft-tissue infection (SSTI) is a common healthcare problem in the pediatric patient population. The incidence of SSTIs has increased profoundly over the past two decades and now represents the seventh most common reason for hospital admission among children [1]. Children, especially those under 3 years of age, appear to be particularly vulnerable to these infections. This represents an increasing financial burden on the healthcare system, with overall costs of hospitalizations for SSTI estimated at \$184 million annually [2].

The majority of SSTIs are caused by Methicillin-resistant Staphylococcus aureus (MRSA). Communityassociated MRSA (CA-MRSA) was first acknowledged in the late 1990s [3] and has skyrocketed in prevalence since that time. The expansion of SSTIs has paralleled the emergence of MRSA, representing an important problem for healthcare systems [4].

Incision and drainage (I\&D) is frequently required for definitive treatment of SSTIs. Most minor infections are

\footnotetext{
${ }^{*}$ Corresponding author.
}

managed successfully by primary care physicians. Many severe SSTIs with abscess formation require surgical consultation. The purpose of this study was to review common characteristics of children requiring surgical referral for I\&D of subcutaneous abscesses.

\section{Methods}

Approval was obtained from the University of Kentucky's Institutional Review Board (IRB 11-0560-P3H). We retrospectively reviewed the medical records of all patients who underwent I\&D of skin and soft tissue infections by the pediatric surgery service at Kentucky Children's Hospital between July 2006 and June 2011. Hospital charts, operative reports, and postoperative clinic notes were examined. Data was collected regarding patient demographics, abscess location, surgical procedures, microbiology cultures, and hospital length of stay (LOS). Demographic data included the age, gender, and race of each patient. The location of the SSTI was classified as head/neck, upper extremities, chest/axilla/back, abdomen, groin/buttock/perineum, or lower extremities. Rates of recidivism were recorded. 
SSTIs with abscess formation were treated operatively with either traditional packing or minimally invasive drainage. Traditional packing (TP) involves open I\&D of the abscess cavity with packing until the wound heals by secondary intention. Minimally invasive (MI) drainage involves small stab incisions, abscess cavity debridement and irrigation, and placement of subcutaneous silastic drains (Figure 1). The drains are removed after several days when the infection has resolved. Prior to the summer of 2010, all abscesses at our institution were treated with traditional packing. During 2010, we transitioned to the MI technique, which has become our preferred method.

\section{Results}

During the six-year study period, 1094 pediatric patients were referred to pediatric surgery for I\&D of SSTIs. These patients ranged in age from seven days to 18.4 years. The average age was 40.7 months (3.4 years), and median age was 20 months (Figure 2). The vast majority of patients $(70.2 \%)$ were less than three-year-old. The study population was $58.1 \%$ female, representing a female: male ratio of 1.39. Most of the children treated were Caucasian (73.6\%), with the remainder being primarily African-American (10.1\%) or Hispanic (3.7\%).

The number of SSTIs requiring surgical drainage increased dramatically during the study period. Only 52 I\&Ds were performed in the 2005-2006 academic year, but numbers increased rapidly thereafter. Over 200 cases have been reported each year since 2008-2009. The dramatic increase in procedures performed is largely attributable to the growing prevalence of MRSA. MRSA was identified from $17 \%$ of culture isolates in 2005-2006; this escalated to $67.5 \%$ in $2006-2007$ and $77 \%$ in $2008-2009$

(Figure 3). Methicillin-sensitive Staphylococcus aureus (MSSA) caused a less significant number of infections (13.8\%). Beta-hemolytic Streptococcus, coagulasenegative Streptococcus, Escherichia coli, and Enteroco-

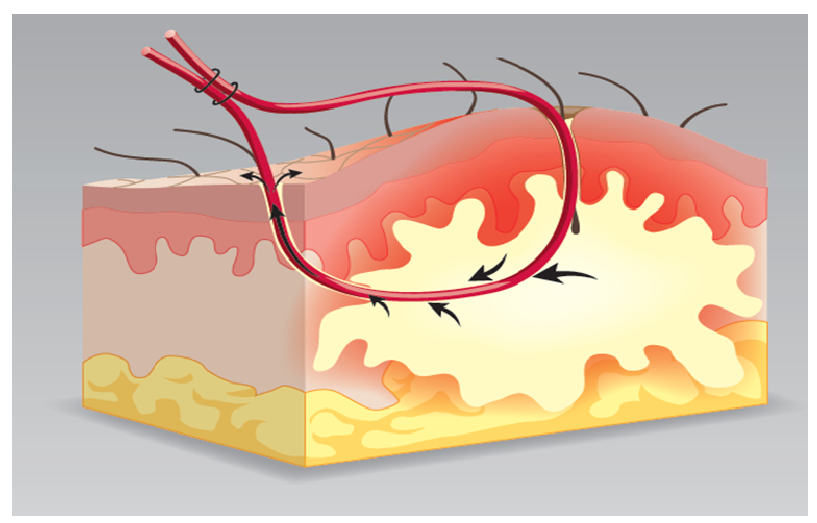

Figure 1. Minimally invasive method of SSTI drainage using silastic drain placement. ccus faecalis were responsible for the remainder of SSTIs.

The vast majority of SSTIs drained (61.2\%) were located on the groin/buttock/perineum area. 20.6\% were found on the lower extremities. The remainder occurred on the abdomen (6.5\%), chest/axilla/back $(6.0 \%)$, head/ neck (7.5\%), and upper extremities (3.3\%). 56 patients (5.1\%) presented with abscesses in multiple locations. Large abscesses sometimes required placement of multiple drains during the operative procedure. For our study, number of drains placed during minimally invasive drainage ranged from one to three silastic drains. The average number placed was 1.3 drains per patient.

The overall average length of hospitalization for children with SSTIs was 2.8 days, with minimum and maximum stays of one and 17 days, respectively. Patients admitted to the surgical service had an average LOS of 2.7 days (minimum one day to maximum ten days), while those admitted to a general pediatric service had an average LOS of 3.1 days (minimum 1 day to maximum 9 days). Pediatric subspecialty service patients averaged a LOS of 4.1 days, ranging from 2 days to 17 days. In general, patients admitted to non-surgical services tended to have longer inpatient hospitalizations. Only $10.4 \%$ of cases during the study period were performed on an outpatient basis. $11.7 \%$ of cases resulted in recidivism. In most instances, this occurred only once; however, one patient presented six different times with skin abscesses requiring I\&D.

\section{Discussion}

The dramatic rise in severe skin and soft tissue infections and the emergence of MRSA as a causative pathogen represent a tremendous public health burden. In the pediatric population, these infections commonly manifest as abscesses requiring surgical incision and drainage, which causes significant difficulty for both patients and caregivers. Costs of treatment includes hospital and operating room charges, medical supplies, antibiotics, follow-up visits, and caregiver's lost income from time off work. Skin infections are often recurrent and are notably difficult to eradicate [2].

Risk factors for SSTIs include age less than 3 years, previous exposure to MRSA, crowding, low socioeconomic status, race, poor hygiene, and moist environments $[4,5]$. Our data confirm these reports as a significant majority of our surgical I\&Ds were performed in children under 2 years of age. Most abscesses in this age group are in the diaper area (groin/buttock/perineum, 61.2\%). We believe that this is because children in diapers have frequent minor skin breaks that allow entrance of skin bacteria into the soft tissues. Furthermore, the diapers provide the ideal warm, moist environment for pathogens to thrive. 


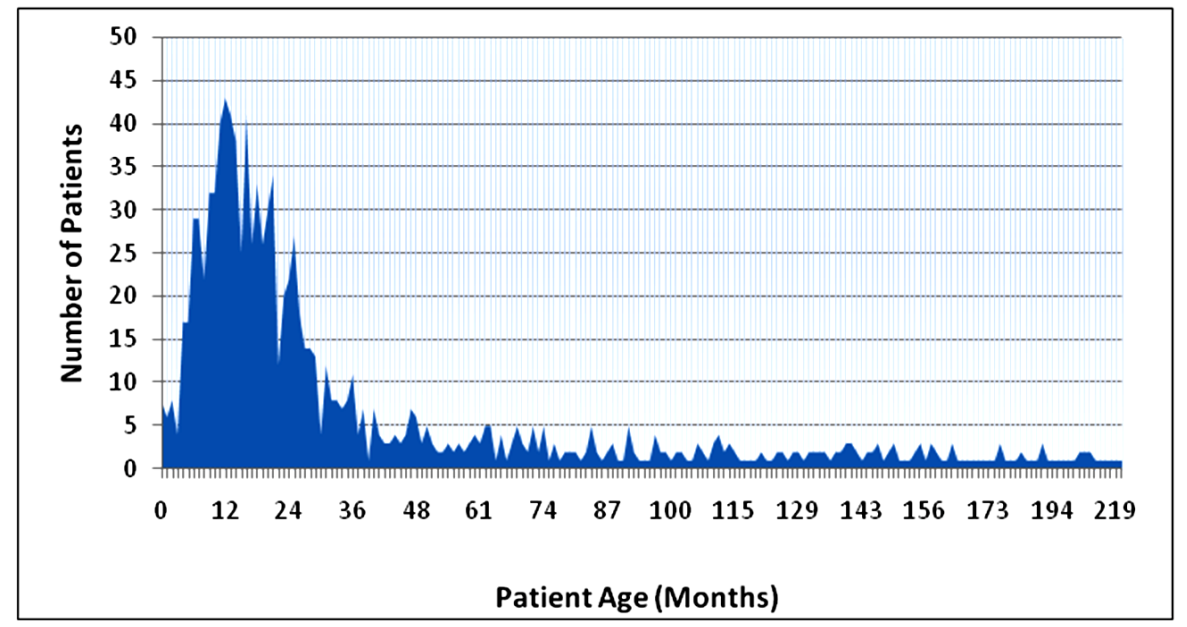

Figure 2. Age distribution of patients referred to pediatric surgeons for drainage of SSTIs.

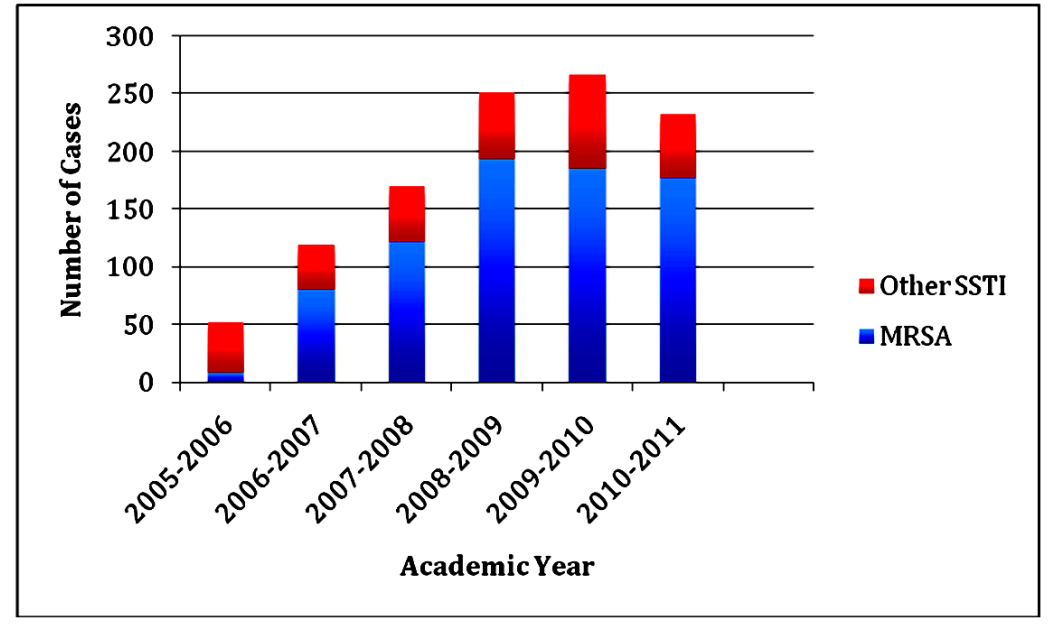

Figure 3. Incidence of SSTIs and MRSA per academic year.

The majority of SSTIs are caused by MRSA [5,6]. Again, this is confirmed in our data, as MRSA represents $70.2 \%$ of the abscesses over the course of our study period. CA-MRSA is the most commonly identified strain. CA-MRSA is distinct from hospital-associated MRSA (HA-MRSA) both genotypically and with respect to the infected population [7]. The increase of MRSA has been previously associated with increasing numbers of severe SSTIs in the pediatric population [5]. MRSA differs from MSSA primarily through the function of Panton-Valentine leukocidin toxin (PVL), a beta-pore forming cytotoxin that attacks soft tissues and creates pores in the membranes of infected cells [8]. MRSA is highly communicable by skin-to-skin contact and outbreaks have been reported in groups such as daycares, prisons, sports teams, and healthcare workers [6].

Our management plan is as follows. Patients should remain NPO until evaluated by the surgical team. If systemic inflammatory signs (fever, malaise, etc.) are present, blood cultures and a complete blood cell count are obtained. A peripheral IV is started for hydration and intravenous antibiotic treatment with either vancomycin or clindamycin is initiated for SSTIs with significant cellulitis. Our institution's antibiogram shows that about $20 \%$ of MRSA isolates are resistant to clindamycin.

The patient is taken to the operating room or procedural sedation suite, and the abscess is drained via the MI technique. The number of drains placed varies based on abscess size and severity (one to three drains typically). This technique, as described previously, results in easier wound care and increased patient and caregiver comfort [9-11]. Furthermore, this technique has been shown to decrease hospital LOS (Draus, unpublished data), and our recidivism rate dropped from $11.7 \%$ to $6.5 \%$. Drains are kept in place until complete resolution of cellulitis and minimal fluid drainage from the wound is noted, often during clinic follow-up approximately 7 - 10 days after the index procedure.

Controversy exists regarding adjunctive antibiotic use after surgical I\&D. For small, simple abscesses, I\&D is 
generally considered sufficient. More complex infections (multiple sites, systemic illness, difficult locations, or patients at extremes of age) often require continued antibiotic therapy [12]. No consensus statement currently guides antibiotic choice and duration of therapy for SSTIs in the pediatric population, and further research is needed to establish a standard of care. Typically, our patients are discharged home on oral sulfamethoxazole/ trimethoprim for several days. Regardless of antibiotic therapy, the most important and definitive treatment of SSTI is drainage, and resolution rarely occurs without surgical intervention [13].

Recidivism is a major problem in these infections, even in patients who are adequately treated with I\&D. About one in ten children from our patient cohort who were treated for SSTI experienced a repeat infection, and a subset of patients experience multiple recurrences. At our institution, patients with multiple abscesses, extremely young patients, and patients with family histories for MRSA abscesses are referred to the Pediatric Infectious Disease service, where they may receive therapy for eradication of MRSA colonization. Adequate I\&D and bacterial eradication therapy can decrease recidivism but fails to prevent all recurrences.

\section{Conclusion}

Our study clearly demonstrates an increase in both SSTIs and MRSA pathology during the study period. Likewise, we demonstrate a clear relationship between patient age and infection site. Identifying the patient populations most at-risk for severe SSTIs will facilitate primary care providers in completing prompt surgical referral. With the growing burden of MRSA SSTIs in the pediatric age group, aggressive treatment is paramount. Our study is limited by its retrospective nature, and by the limited data set collected. Additional research may allow us to identify subsets of patients at higher risk for recidivism or those at risk for more severe infections. Additionally, further characterization of these patients will allow more appropriate surgical referral patterns and treatment algorithms to reach the majority of pediatric care providers.

\section{REFERENCES}

[1] B. Friedman, T. Berdahl, L. A. Simpson, et al., “Annual Report on Health Care for Children and Youth in the United States: Focus on Trends in Hospital Use and Quality,” Academic Pediatrics, Vol. 11, No. 4, 2011, pp. 263-279. doi:10.1016/j.acap.2011.04.002

[2] T. Lautz, M. Raval and K. Barsness, "Increasing National Burden of Hospitalizations for Skin and Soft Tissue Infections in Children," Journal of Pediatric Surgery, Vol. 46, No. 10, 2011, pp. 1935-1941.

\section{doi:10.1016/j.jpedsurg.2011.05.008}

[3] Centers for Disease Control, "Four Pediatric Deaths from Community-Acquired Methicillin-Resistant Staphylococcus aureus-Minnesota and North Dakota, 1997-1999," Journal of the American Medical Association, Vol. 283, No. 12, 1999, pp. 1123-1125.

[4] C. Frei, B. Makos, K. Daniels, et al., "Emergence of Community-Acquired Methicillin-Resistant Staphylococcus aureus Skin and Soft Tissue Infections as a Common Cause of Hospitalization in United States Children,” Journal of Pediatric Surgery, Vol. 45, No. 10, 2010, pp. 1967-1974. doi:10.1016/j.jpedsurg.2010.05.009

[5] A. Shapiro, S. Raman, M. Johnson, et al., "CommunityAcquired MRSA Infections in North Carolina Children: Prevalence, Antibiotic Sensitivities, and Risk Factors," North Carolina Medical Journal, Vol. 70, No. 2, 2009; pp. 102-107.

[6] A. Pickett, M. Wilkinson, M. Menoch, et al., "Changing Incidence of Methicillin-Resistant Staphylococcus aureus skin Abscesses in a Pediatric Emergency Department,” Pediatric Emergency Care, Vol. 25, No. 12, 2009, pp. 831-834. doi:10.1097/PEC.0b013e3181c06230

[7] A. McCullough, M. Seifried, X. Zhao, et al., "Higher Incidence of Perineal Community Acquired MRSA Infections among Toddlers,” BMC Pediatrics, Vol. 11, 2011, p. 96. doi:10.1186/1471-2431-11-96

[8] G. Lina, Y. Piemont, F. Godail-Gamot, et al., "Involvement of Panton-Valentine Leukocidin-Producing Staphylococcus aureus in Primary Skin Infections and Pneumonia,” Clinical Infectious Diseases, Vol. 29, No. 5, 1999; pp. 1128-1132. doi:10.1086/313461

[9] W. F. McNamara, C. E. Hartin Jr., M. A. Escobar, et al., "An Alternative to Open Incision and Drainage for Community-Acquired Soft Tissue Abscesses in Children," Journal of Pediatric Surgery, Vol. 46, No. 3, 2011, pp. 502-506. doi:10.1016/j.jpedsurg.2010.08.019

[10] S. Tsoraides, R. Pearl, A. Stanfill, et al., "Incision and Loop Drainage: A Minimally Invasive Technique for Subcutaneous Abscess Management in Children," Journal of Pediatric Surgery, Vol. 45, No. 3, 2010, pp. 606- 609. doi:10.1016/j.jpedsurg.2009.06.013

[11] A. Ladd, M. Levy and J. Quilty, "Minimally Invasive Technique in Treatment of Complex, Subcutaneous Abscesses in Children," Journal of Pediatric Surgery, Vol. 45, No. 7, 2010, pp. 1562-1566. doi:10.1016/j.jpedsurg.2010.03.025

[12] A. Hersh, H. Chambers, J. Maselli, et al., "National Trends in Ambulatory Visits and Antibiotic Prescribing for Skin and Soft Tissue Infections," Archives of Internal Medicine, Vol. 168, No. 14, 2012, pp. 1585-1591. doi:10.1001/archinte.168.14.1585

[13] C. Liu, A. Bayer, S. Cosgrove, et al., "Clinical Practice Guidelines by the Infectious Diseases Society of America for the Treatment of Methicillin-Resistant Staphylococcus aureus Infections in Adults and Children: Executive Summary,” Clinical Infectious Diseases, Vol. 52, No. 3, 2011, pp. 285-292. doi:10.1093/cid/cir034 